\title{
Clinical study or research activity?
}

Sam Eagle, DVM, a veterinary neurologist, was the deal-maker at the Great Eastern University veterinary school, a USDAregistered and NIH/OLAW-Assured institution that included all vertebrate animals, no matter the funding source for the research in which they were used, in its Assurance. Eagle had friends in all the nearby pharmaceutical companies and human hospitals, and so it was not surprising when he convinced Southedge Hospital, a tertiary care facility, to agree to carry out computed axial tomography (CAT) scans on dogs that came through the veterinary school's small animal clinic and were suspected of having had a stroke. There would be no charge to the animals' owners, as long as they also consented to have periodic functional magnetic resonance imaging (fMRI) studies on their dogs at Southedge, also at no charge to the owners. The initial CAT scan was diagnostic, but the fMRI studies were for Eagle's research and were paid for by Eagle's Everice Foundation grant.

The IACUC at Great Eastern was fully aware of Eagle's collaboration with Southedge and willingly allowed the veterinary school's Clinical Trials Committee to review and oversee Eagle's work at the human hospital. The rationale from the IACUC was that the animals were privately owned and the CAT scans were for diagnostic purposes. As for the fMRI studies, the IACUC took the position that they may have clinical utility, although Eagle proudly advertised it as his research. On the other hand, the school's veterinarians were frustrated that there was no IACUC oversight for exactly the same reason:
Eagle readily stated that it was part of his research. Furthermore, the veterinarians did not consider the anesthesia needed for the fMRI studies to be innocuous; rather, they saw it as a potentially dangerous aspect of a research activity that had no direct benefit to the dogs. Eagle neither agreed nor disagreed with them. He said that it was just a matter of personal opinion and that he had no opinion at all on the issue.

What do you think? Is Eagle's work with privately owned animals a clinical study that was really designed for research purposes, or is it a pure clinical study as the Great Eastern IACUC claims? In the former instance, is IACUC approval needed? If so, should approval come from the veterinary school's IACUC, or does Southedge Hospital need to be registered with the USDA and have its own IACUC?

\section{RESPONSE}

\section{IACUC should review}

\section{Lara A. Weaver, DVM \& \\ Brian J. Moore, MS, DVM}

A clinical trial is usually done to determine the safety or efficacy of a diagnostic tool or treatment designed to be of benefit for a clinical condition in the research subject. Ideally, the procedure or drug should address the specific medical condition of and have some direct benefit to the animals enrolled. In this scenario, the dogs were chosen to participate in this study on the basis of clinical indications that they had suffered a stroke. As CAT scans are diagnostically useful in this scenario, this portion of the work could legitimately be considered a clinical procedure and be covered by the Clinical Trials Committee.

The subsequent fMRIs do not have any diagnostic or therapeutic merit, however, and the associated anesthesia poses a real risk to the dogs. The fMRI portion of this study should be considered research, just as Eagle has advertised. Although the Animal Welfare $\mathrm{Act}^{1}$ does not explicitly address the use of privately owned animals in research, the dogs in this study would meet the Animal Welfare Regulations' definition of "animal." The Public Health Service requires research studies using species listed in the university's Animal Welfare Assurance to have IACUC approval; therefore, regardless of the USDA's position in this situation, Great Eastern's Public Health Service Assurance would dictate that this research be covered by an IACUC protocol ${ }^{2}$. A detailed informed consent document explaining the risks to the owners should also be evaluated during the IACUC review process.

As for the question of which IACUC should review this protocol, again Great Eastern has elected to include "all vertebrate animals, no matter the funding source" in its Assurance, requiring the Great Eastern IACUC to review this protocol. Aside from the regulatory requirements, it may be in the best interests of both the clients and the veterinary school to have Great Eastern's IACUC review the fMRI portion of the study. The Great Eastern IACUC would likely include more veterinarians and should be more accustomed to reviewing protocols of this nature than a committee at a human hospital would be. The review at the veterinary school would also enable the school to have more input in and oversight of the process, which is beneficial to both the animals and the institution. In the event that Southedge does not already have an Assurance or IACUC in place, using the Great Eastern IACUC to review the protocol would also prove to be a more convenient option. Great Eastern could then include Southedge under its Assurance for the purposes of this study and could carry out semiannual inspections of the facility during the time period in which the studies take place.

This unique scenario raises questions that may not have clear-cut answers. In addition to the points raised above, there are also regulatory compliance issues relating to 